\title{
Dans le cône d'ombre de la crise : historiens et histoires des années quatre-vingt en Italie
}

Nel cono d'ombra della crisi: storici e storie degli anni Ottanta in Italia In the "dark spot " of the crisis: historians and histories of the eighties in Italy

\section{Andrea Rapini}

\section{OpenEdition}

\section{Journals}

Édition électronique

URL : http://journals.openedition.org/cei/330

DOI : $10.4000 /$ cei.330

ISSN : 2260-779X

\section{Éditeur}

UGA Éditions/Université Grenoble Alpes

Édition imprimée

Date de publication : 15 mars 2012

Pagination : 19-45

ISBN : 978-2-84310-222-6

ISSN : 1770-9571

\section{Référence électronique}

Andrea Rapini, «Dans le cône d'ombre de la crise : historiens et histoires des années quatre-vingt en Italie ", Cahiers d'études italiennes [En ligne], 14 | 2012, mis en ligne le 15 septembre 2013, consulté le 28 mars 2021. URL : http://journals.openedition.org/cei/330 ; DOI : https://doi.org/10.4000/cei.330 


\title{
DANS LE CÔNE D'OMBRE DE LA CRISE : HISTORIENS ET HISTOIRES DES ANNÉES QUATRE-VINGT EN ITALIE ${ }^{\mathrm{I}}$
}

\author{
Andrea Rapini \\ Université de Modène et Reggio d'Émilie
}

[...] non basta più capire le cose nuove che sono successe e che succedono, non basta piu aggiornare le conoscenze, occorre qualcosa di più, occorre un diverso modo di pensare, di rapportare la mente alla realtà [...].

Se si vuole salvare la stessa idea del cambiamento, la stessa Gerusalemme, bisogna rileggere il presente, scorgere in esso il futuro, non separare più il domani dall'oggi, riscoprire Gerusalemme attorno a noi, dentro di noi.

Vittorio FOA ${ }^{2}$

\section{Pour une histoire de l'histoire du temps présent}

L'historiographie italienne a désormais réussi à construire une réflexion aboutie sur le mouvement de 1968. Bien qu'il reste encore un champ très vaste pour des recherches et des approfondissements, surtout en ce qui concerne la comparaison internationale de l'événement, on peut dire qu'il existe suffisamment de recherches locales et de synthèses pour insérer de façon significative cette période dans la longue durée de l'histoire nationale. Les volumes de Guido Crainz et Marica Tolomelli sont un miroir efficace de ce que l'on a pu dire sur ce sujet ${ }^{3}$.

I. Cet article a été rédigé en 20Io. Toutes les citations italiennes y ont été traduites directement par nos soins.

2. La Gerusalemme rimandata. Domande di oggi agli inglesi del primo Novecento, Turin, Einaudi, 1985, p. 366.

3. G. Crainz, Il paese mancato: dal miracolo economico agli anni Ottanta, Rome, Donzelli, 2005 [2003]; M. Tolomelli, Movimenti collettivi nell'Europa di fine anni '6o: guida allo studio dei movimenti in Italia, 
Cependant, la compréhension de la décennie suivante apparaît plus difficile. Si d'un côté, il existe de nombreux apports sociologiques, politiques, économiques et que les articles journalistiques ainsi que les mémoires des militants politiques souvent issus de la lutte armée abondent, d'un autre côté, il manque encore une reconstruction d'ensemble de la période ${ }^{4}$. La décennie reste floue et sa complexité finit souvent par entraîner une certaine confusion, à cause de la surexposition d'une seule de ses parties. Prenons l'exemple de la fameuse formule « les années de plomb », qui aspire toute innovation politique, culturelle et sociale dans le tourbillon obscur du terrorisme. Cette expression est déjà problématique en soi, car elle annule les différences entre les idéologies, la morphologie et la pratique de la violence de la droite et de la gauche, en plus d'ignorer l'autoreprésentation des protagonistes. Coincées entre la violence politique, la répression de l'État et la crise économique, les années soixante-dix échappent encore à une réflexion historiographique pondérée et restent un terrain fertile pour des plus incursions excessives dans les usages publics de l'histoire.

Les années quatre-vingt apparaissent enfin comme une véritable " terre étrangère ", ou bien comme une sorte de "frontière " qui reste totalement à conquérir. Au-delà des métaphores, les études analytiques sur des questions isolées aussi bien que les œuvres d'ensemble font aujourd'hui défaut, à quelques exceptions près. Tel est le cas, par exemple, des récents travaux sur Bettino Craxi, qui est, cela va de soi, l'une des personnalités clé de cette périodes. Toutefois, cette décennie représente un problème historiographique de grande envergure, du moment où c'est principalement de cette période qu'émane l'Italie d'aujourd'hui.

Dans ce qui va suivre, on passera en revue certaines interprétations historiques des années quatre-vingt, choisies pour la plupart au sein du vaste panel de synthèses de l'Italie républicaine, qui représentent les tentatives les plus abouties de situer les années 1980 dans les cycles longs de l'histoire précédente. La sélection de ce matériel ne prétend aucunement être exhaustive. Notre choix dépend uniquement de la volonté de présenter un panorama complexe et diversifié des différentes possibilités interprétatives offertes par l'historiographie.

Germania e Francia, Bologne, Patron, 2002; Id., Il sessantotto: una breve storia, Rome, Carocci, 2008.

4. Voir L'Italia repubblicana nella crisi degli anni Settanta: atti del ciclo di convegni, Rome, nov.-déc. 20oI, 4 vol., Rubbettino, Soveria Mannelli, 2003; A. De Bernardi, V. Romitelli et C. Cretella (dir.), Gli anni Settanta: tra crisi mondiale e movimenti collettivi, Bologne, Archetipo, 2009.

5. S. Colarizi et M. Gervasoni, La cruna dell'ago: Craxi, il partito socialista e la crisi della Repubblica, RomeBari, Laterza, 2005. 
Un préambule est d'abord nécessaire pour saisir de quelle manière l'expression " années quatre-vingt " prend un sens historiographique éloigné du sens commun. Les décennies des historiens sont des constructions conceptuelles qui divergent des décennies du calendrier, lesquelles, par convention, procèdent de façon ordonnée et immuable, de dix ans en dix ans. L'objet des historiens, dont je vais m'occuper à présent, est formé par des problèmes et des processus qui traversent la décennie conventionnelle en avant et en arrière, sans disparaître avec elle. Les œuvres prises en compte sont des exemples d'histoire du temps présent, où l'immersion de l'historien dans le flux des événements étudiés influence inévitablement sa pratique historique, la rendant particulièrement apte à la production de schémas de compréhension pour le présent ${ }^{6}$. Cependant, le chercheur y est aussi beaucoup plus exposé au risque d'être dominé par ses passions.

Mon étude se basera sur le travail de six historiens : Paul Ginsborg, Silvio Lanaro, Enzo Santarelli, Pietro Scoppola, Gaetano Quagliariello et Guido Crainz. Toutes ces interprétations des années quatre-vingt sont comprises dans l'arc chronologique 1989-2009. Vingt années qui coïncident avec une phase de crise et de transition de l'Italie, d'un système politique à un autre, d'un apparat constitutionnel à un autre, en vertu de l'émergence d'un phénomène international d'une part, et d'un phénomène interne d'autre part : l'implosion du communisme - la fatidique année 1989 - et l'enquête judiciaire "Mani pulite ". Dans un tel climat, l'impression de traverser une sorte de " no man's land " est si répandue qu'elle autorise l'emploi des néologismes (d'influence française) « Première République » et "Seconde République » pour indiquer la fracture survenue ainsi que la période historique en cours. Ces néologismes, répandus dans le milieu journalistique et utilisés de façon superficielle par certains historiens, ne sont absolument pas neutres, car ils font la promotion d'une réforme de la constitution formelle qui devrait s'adapter à une altération de la constitution matérielle qui a déjà eu lieu7. Dans les pages qui suivantes, on cherchera à mettre en lumière le fait que toutes les interprétations sont profondément marquées :

I. par la biographie de l'historien, par sa culture historique et politique;

6. F. Bédarida, Histoire, critique et responsabilité, Bruxelles, Éd. Complexe, 2003.

7. L'expression "Seconde République " est utilisée pour la première fois par les Fascistes d'action révolutionnaire, une organisation néofasciste qui en 1947 promeut « l'instauration de l'ordre fasciste en Italie avec la II ${ }^{e}$ République sociale italienne ». C'est Randolfo Pacciardi qui l'utilisera ensuite pour indiquer une proposition de type présidentialiste. 
2. par la situation chronologique de l'interprétation à l'intérieur de la phase de crise et de transition (le moment historique où écrit l'historien);

3. par la définition de crise fournie par l'historien, à partir de laquelle on en recherche les racines dans le passé.

Ce que l'on tente donc de montrer est le caractère socialement, institutionnellement et politiquement situé de l'historien ${ }^{8}$. Ce dernier doit être expulsé du paradis illusoire de l'extra-temporalité et de l'indépendance absolue, sans pour autant tomber dans un relativisme stérile, dans une obscurité au sein de laquelle les différences entre narration scientifique et narration fictionnelle se réduisent jusqu'à disparaittre9. L'historien du temps présent se situe dans la tension entre les vérités partiales - obtenues à travers des preuves et des procédures reconnues par la communauté scientifique - et les conditionnements qui émanent de l'appartenance à l'histoire qu'on raconte $^{\mathrm{Io}}$. Cependant, à bien y regarder, ce raisonnement touche au cœur de la pratique historique. Pour ne pas subir ces conditionnements inconsciemment et en être prisonniers, il ne nous reste qu'à souhaiter une connaissance des conditions de la connaissance ${ }^{\text {II }}$.

\section{Au bord du gouffre}

Paul Ginsborg est un historien anglais arrivé en Italie en 1992, après avoir enseigné à la faculté de sciences sociales et politiques du Churchill College de Cambridge. Il est actuellement professeur d'histoire de l'Europe à Florence. Sa pratique historique, influencée par le marxisme anglo-saxon des années soixante et soixante-dix, s'attache tout particulièrement aux diverses articulations des formes culturelles et à leur rapport avec l'analyse de la société. Ses références sont Raymond Williams, Edward Thompson, Christopher Hill et Eric Hobsbawm.

En 1989, Ginsborg publie dans une des plus importantes maisons d'édition italiennes (Einaudi), appartenant à Silvio Berlusconi depuis 1994, la

8. Voir M. De Certeau, L'écriture de l'histoire, Paris, Gallimard, 1975 et R. Chartier, Au bord de la falaise. L'histoire entre certitudes et inquiétude, Paris, Albin Michel, 2008 [1998].

9. C. Ginzburg, Rapporti di forza. Storia, retorica, prova, Milan, Feltrinelli, 2000.

IO. H. Rousso, "L'histoire du temps présent, vingt ans après ", dans L'histoire du temps présent, hier et aujourd'hui, Bulletin de l'IHTP, $\mathrm{n}^{\circ} 75$, 2000. Sur la construction de la notion d'histoire du temps présent en Italie dans l'après-guerre, voir V. Galimi, "L'histoire du temps présent en Italie ", La revue pour l'histoire du CNRS, nº 9, 2003 (<http://histoire-cnrs.revues.org/563>).

II. Voir P. Bourdieu, Le sens pratique, Paris, Les Éditions de Minuit, I989. 
Storia d'Italia dal dopoguerra ad oggi, suivie de L'Italia del tempo presente. Famiglia, società civile, Stato 1980-1996 ${ }^{12}$.

Ces deux volumes représentent encore aujourd'hui la reconstruction la plus complète de la trajectoire italienne de la fin de la seconde guerre mondiale au milieu des années quatre-vingt-dix. Pour compléter cette œuvre de grande envergure, d'autres travaux suivront, nés d'un ensemble d'activités politiques et civiles, auxquelles Ginsborg se consacre durant les années 2000 : Berlusconi. Ambizioni patrimoniali in una democrazia mediatica ${ }^{13}$; Il tempo di cambiare. Politica e potere della vita quotidiana ${ }^{14}$ et La democrazia che non c'è ${ }^{15}$.

Si l'on exclut le volume de Giuseppe Mammarella, paru en 1970 et puis réédité à plusieurs reprises ${ }^{16}$, Storia d'Italia dal dopoguerra ad oggi est la première synthèse sur l'histoire de l'Italie républicaine. Les années suivantes, cette œuvre subira la concurrence de nombreux produits similaires. On peut d'ailleurs parler de l'inauguration d'une sorte de genre, dont la fortune est sans aucun doute à attribuer en grande partie à l'appétit des éditeurs. Le volume, qui a été apprécié aussi bien par la critique historiographique que par un public plus vaste, presse comprise, constitue certainement un moment clé pour l'historien anglais, qui, grâce à la notoriété acquise, est devenu par la suite un terme de référence respecté à l'intérieur du champ historiographique italien, mais aussi en dehors. Bien que la décennie ne soit pas encore achevée et que la crise du début des années quatre-vingt-dix soit encore toute récente, le dernier chapitre du livre (xI) est consacré à l'Italie des années 80 . Il convient d'en faire un court résumé, car ce thème sera abordé dans le livre suivant du même auteur ${ }^{17}$. De plus, les thèses qui y sont élaborées seront reprises par d'autres d'auteurs ${ }^{18}$. Ginsborg propose une analyse structurée de l'économie, de la société, de la famille, des valeurs, de la politique, mais aussi une interprétation d'ensemble. Tandis que les

I2. Turin, Einaudi, 1998.

I3. Turin, Einaudi, 2003.

I4. Turin, Einaudi, 2004 .

I5. Turin, Einaudi, 2006. En 2002, durant le second gouvernement Berlusconi, Ginsborg fonde à Florence le Laboratoire pour la démocratie, qui souhaite d'un côté " organiser une opposition intransigeante " à l'entrepreneur-président du Conseil, et d'un autre côté d' " entamer un long processus de révision critique de la démocratie ", en rénovant la culture politique de la Gauche. Plus récemment, après la disparition des partis de Gauche du Parlement italien lors des législatives de 2008, il tente de réunifier les multiples courants de la gauche italienne, en proie à une sorte de "folie centrifuge ", ou mieux à un cupio dissolvi masochiste. Voir : <http:// www.officinapolitica.it/ritagli/ginsborg.pdf>.

16. L'Italia dopo il fascismo: 1943-1968, Bologne, Il Mulino, 1970.

17. L'Italia del tempo presente. Famiglia società civile, Stato 1980-1996, ouvr. cité.

I8. G. Crainz, Autobiografia di una Repubblica. Le radici dell'Italia attuale, Rome, Donzelli, 2009; G. De Luna, Le ragioni di un decennio, Milan, Feltrinelli, 2009. 
quatre premiers secteurs de l'Italie connaissent un dynamisme intense, la politique et l'État se démènent dans l'immobilisme, incapables qu'ils sont d'orchestrer le changement à travers un programme de réformes. Si la décennie précédente est résumée par l'expression L'époque de l'action collective (cap. IX), les années quatre-vingt se déroulent au contraire à l'enseigne de l'action individuelle et prennent de l'ampleur sur la défaite d'une mobilisation de masse : cette fameuse année 1980 chez Fiat, où le licenciement des militants les plus radicaux de l'usine, après trente-cinq jours de lutte, marque symboliquement la transition historique ${ }^{19}$. La fin d'une époque est justement le paragraphe qui tourne la page des années soixante-dix. Dans Changement des valeurs, qui est le nom du dernier paragraphe du libre, Ginsborg dresse un portrait parfait de la nouvelle Italie :

La grande transformation de l'Italie, alors, a été de s'adapter au modèle de modernité apparu pour la première fois à l'époque du « miracle économique », un modèle à forte influence américaine, extrêmement contesté entre I968 et 1973, mais qui semble avoir trouvé son âge d'or dans les années $80^{20}$.

Le modèle auquel l'auteur se réfère se concentre sur la diffusion de la consommation individuelle et sur son pouvoir de surdétermination des styles de vie, des besoins et des désirs. Toutefois, sans prendre un ton apocalyptique, fidèle à la leçon d'Albert Hirschmann, Ginsborg rappelle que la société de consommation contient la satisfaction aussi bien que son contraire, c'est-à-dire la désillusion ${ }^{21}$. Il se demande donc si « les valeurs des années quatre-vingt dureront, ou bien si des visions alternatives pourront encore avoir un rôle, même minimal, dans l'histoire de l'Italie républicaine $^{22}$ ». Les vingt années suivantes (1989-2009) se chargeront de donner une réponse à ce questionnement.

En I992, en même temps que le début de l'enquête judiciaire "Mani pulite " qui fera basculer la soi-disant " Première République ", l'une des reconstructions les plus appréciées de l'histoire italienne d'après-guerre voit le jour : Silvio Lanaro, Storia dell'Italia repubblicana. Dalla fine della guerra agli anni novanta, Marsilio, Venezia (Prix Acqui Storia 1993 et Prix de la Société italienne pour l'étude de l'histoire contemporaine-Sissco I993). L'auteur, qui durant sa jeunesse a traversé la New Left italienne et qui évolue

19. Sur ce sujet, voir M. Revelli, Lavorare in Fiat, Milan, Garzanti, I989.

20. P. Ginsborg, Storia d'Italia dal dopoguerra ad oggi, ouvr. cité, p. 575-576.

2I. A. Hirschmann, Shifting involvements: private interest and public action, Princeton, Princeton University Press, I982.

22. P. Ginsborg, Storia d'Italia dal dopoguerra ad oggi, ouvr. cité, p. 576. 
encore aujourd'hui dans la mouvance de la Gauche réformiste, est professeur d'histoire contemporaine à l'université de Padoue. En tant que chercheur, il s'est occupé du rapport entre idéologies et société dans la Vénétie rurale, mais aussi de nationalisme, de cultures du travail et des caractères originels de la modernisation italienne ${ }^{23}$.

Le livre de Lanaro se situe au bord du gouffre : d'un côté, il y a la sensation très forte que le pays est désormais dos au mur, d'un autre on a du mal à imaginer une solution au problème. Dans le dernier paragraphe - Etre normaux - l'auteur donne sa définition de la crise : "crise du système politique, tout comme crise de la constitution matérielle, en d'autres termes, il ne s'agit pas d'une crise de la constitution formelle». Il faudrait en chercher les origines dans l'occupation de tous les centres de pouvoir de la part des partis et dans " une tendance à nuire à la collectivité dérivant de la paralysie, du non-choix, de l'inaction et de la nullité opérationnelle que provoquent toujours la dépendance des institutions des intérêts ${ }^{24}$ ».

Comment en sortir? Au-delà des exceptionnalismes, il faudrait, selon Lanaro, retrouver la force de gouverner « normalement ». Bref, il faudrait qu'un retour à la norme se produise, mais sans payer un prix trop élevé. Cette définition de la crise et la fin du livre sont aussi alarmistes que prescriptifs, dans la mesure où, dans le concept vague de normalité, réside la volonté implicite de conduire l'Italie vers d'autres modèles de démocratie, souvent anglo-saxons, modèles qui sont constamment au centre des discussions dans l'Italie du début des années quatre-vingt-dix. Le ton de Lanaro porte les stigmates de la conjoncture. Le mal-être profond dans lequel est plongé l'Italie de 1989 à 1992, avant l'implosion du système politique, rend difficile l'élucidation des caractères de la crise. Mais ce mal-être empêche surtout le temps présent de se fondre dans une durée plus ample et emprisonne l'auteur dans le souffle moribond du système politique. La reconstruction des années quatre-vingt qui en découle est très fragile. On les retrouve dans un paragraphe - La saison des xillets - où le manque d'analyse de la société et de l'économie est compensé par l'omniprésence de Bettino Craxi. Contrairement à Ginsborg, qui en plus de présenter les propositions politiques du secrétaire du PSI comme dénuées de toute stratégie organique en ce qui concerne les réformes, critique durement sa présidence du Conseil (1983-1987), qui aurait été plus utile au PSI qu’à l'Italie,

23. Parmi ses œuvres principales : Società e ideologie nel Veneto rurale: I866-1898, Rome, Edizioni di storia e letteratura, 1976; Nazione e lavoro. Saggio sulla cultura borghese in Italia (I870-1925), Venise, Marsilio, I979; L'Italia nuova. Identità e sviluppo (I86I-I988), Turin, Einaudi, I988; Patria: circumnavigazione di un'idea controversa, Venise, Marsilio, 1996.

24. S. Lanaro, Storia dell'Italia repubblicana. Dalla fine della guerra agli anni novanta, ouvr. cité, p. 452-453. 
Lanaro donne un jugement opposé, en s'intéressant de près au lien entre la fin et les moyens. Craxi est porteur d'un « dessein très clair " basé sur trois points :

I. Redonner une identité et une image au socialisme italien, en l'arrachant à l'influence marxiste et en l'insérant dans une tradition mutualiste, labouriste, humanitaire, qui a été mise à mal par la cohabitation avec le " grand frère ${ }^{25}$ ";

2. Créer un pôle laïc fort, avec une aptitude décisionnelle, capable de s'insérer au cœur même du pouvoir et de mettre à mal le monopole de la démocratie chrétienne;

3. Affirmer l'orgueil national, en s'émancipant de la dépendance envers les Américains.

Si en ce qui concerne ces "projets précis", le bilan du gouvernement Craxi est " nettement positif ", Lanaro met également en évidence le côté obscur de cette expérience :

Il a complètement démantelé son parti, en étouffant toute velléité de débat et de démocratie interne [...]. Il a entretenu des liaisons dangereuses et protégé des personnages inqualifiables. [...] Il a montré de l'exaspération et de l'indifférence pour [...] la diffusion à un rythme exponentiel de vols, de malversations, d'escroqueries et d'extorsions dans lesquels nombre de ses collaborateurs étaient impliqués. [...] Il a favorisé le phénomène de "l'arrivisme » [...] en le prenant pour une conséquence physiologique de la modernité2 ${ }^{2}$.

\section{Au fond du gouffre}

Les deux années 1992-1994 comptent probablement parmi les plus dramatiques de toute l'histoire républicaine. L'enquête de la magistrature milanaise nommée "Mani pulite » touche et renverse tous les partis de "l'arc constitutionnel ", à savoir tous les partis qui ont écrit la Constitution de I948. Il s'agit d'un véritable tremblement de terre, qui provoque la décomposition provisoire du système de pouvoir fondé sur l'alliance de trente ans entre le parti socialiste (PSI) et la démocratie chrétienne (DC) ${ }^{27}$. En plus de la désagrégation de la classe dirigeante, on est confrontés aux problèmes posés par une situation monétaire des plus critiques pour la devise italienne,

25. Ibid., p. 448.

26. Ibid., p. 450.

27. Le PCI avait déjà décidé de changer son nom en I99i (PDS). 
la lire, à un déficit public dramatique et aux difficultés quotidiennes des classes moyennes. Tout aussi graves sont les conséquences sociales, qu'on peut observer à travers le rapport conflictuel entre les travailleurs et leurs représentants syndicaux : le 22 septembre 1992, le secrétaire du plus grand syndicat italien (CGIL) Bruno Trentin est agressé avec des œufs et des boulons par des jeunes et des militants furibonds pendant un rassemblement à Florence ${ }^{28}$.

Les œuvres qui ont été écrites au fond du gouffre - bien que publiées un peu plus tard pour des besoins éditoriaux évidents - situent la crise non seulement dans le cycle bref ayant succédé à 1989, mais également dans le cycle long post-1945: Enzo Santarelli, Storia critica della Repubblica. LItalia dal 1945 al $1994^{29}$ et Pietro Scoppola, La Repubblica dei partiti. Evoluzione e crisi di un sistema politico 1945 -1996 ${ }^{30}$.

Ex-résistant pendant la guerre de Libération, "militant de la gauche avec des positions communistes ${ }^{31}$ " et professeur d'histoire contemporaine à l'université d'Urbino, Enzo Santarelli (1922-2004) peut être défini comme un intellectuel organique du parti communiste italien (PCI) jusqu'à sa dissolution entre 1989 et 1991. En examinant les titres de certains livres, on observe la passion politique qui l'a constamment guidé : La rivoluzione femminile $^{32}$, Il socialismo anarchico in Italia ${ }^{33}$, La revisione del marxismo in Italia $^{34}$, Storia del movimento e del regime fascista ${ }^{35}$, Pietro Nenni ${ }^{36}$, Profilo del berlusconismo ${ }^{37}$.

Lorsque Santarelli publie la Storia critica della Repubblica, l'expression gramscienne intellectuel organique, si centrale dans l'histoire culturelle et politique italienne, est devenue imprononçable dans le champ académique comme dans le champ médiatique, à cause de la vague de droite ayant suivi la chute du mur de Berlin ${ }^{38}$. De plus, Santarelli est désormais un professeur à la retraite, dont la capacité à conditionner la reproduction du

28. Pour une chronologie détaillée des événements, voir :

$<$ http://www.cattaneo.org/index.asp? $\mathrm{I}_{\mathrm{I}}=\operatorname{archivi} \& \mathrm{l}_{2}=$ crono $>$.

29. Milan, Feltrinelli, 1996.

30. Bologne, il Mulino, 1997 [1991].

3I. Voilà ce que disent les notes biographiques en quatrième de couverture de Storia critica della Repubblica, ouvr. cité.

32. Parme, Guanda, I950.

33. Milan, Feltrinelli, 1973.

34. Milan, Feltrinelli, 1964.

35. Rome, Editori Riuniti, 1967.

36. Turin, Utet, 1988.

37. Rome, Datanews, 2002.

38. Sur la transformation de l'intellectuel : A. Asor Rosa, Il grande silenzio: intervista sugli intellettuali, RomeBari, Laterza, 2009. 
milieu universitaire est presque nulle ${ }^{39}$. Enfin, il sera utile de rappeler une donnée générationnelle : Santarelli est un représentant de la première génération d'historiens de l'après-guerre. À cheval entre les années soixante-dix et quatre-vingt, la seconde génération d'historiens, provenant en grande partie des mouvements des jeunes, soumet à une critique sévère aussi bien les méthodes que les résultats de leurs maîtres plus anciens, pour se distinguer et s'affirmer sur un plan historiographique, politique, académique et générationnel ${ }^{40}$. Tout ceci pour dire qu'au début des années I990, une grande partie des historiens considère comme désuètes les références culturelles de Santarelli, et en particulier Gramsci. L'auteur de la Storia critica della Repubblica est donc un intellectuel dominé. Pour cette raison, son œuvre a sans doute un impact moindre par rapport aux deux synthèses mentionnées auparavant, indépendamment de ses mérites ou de ses limites intrinsèques ${ }^{41}$. Il suffit d'arriver à la page quatorze de la Note introductive pour se faire une idée de la nature du volume :

Ces pages veulent être un essai d'interprétation historique basé sur l'autocritique, provenant d'une expérience collective. Une exploration effectuée sur la base de la crise actuelle $^{42}$.

On note la superposition dans la même personne des rerum gestarum et de l'historia rerum gestarum. De plus, la crise est envisagée comme une perspective d'où l'on peut observer un parcours d'un demi-siècle. Mais quelle crise? Les explications institutionnelles et politiciennes centrées exclusivement sur "Tangentopoli ${ }^{43}$ " et sur le système politique sont insuffisantes selon Santarelli. Il faut plutôt prendre en compte un " ensemble de questions » qui provoquent de multiples réponses dans la courte comme dans la longue durée : la désagrégation du modèle de développement sur lequel se basait l'Occident pendant l'après-guerre; les limites de l'hégémonie américaine internationale et de son influence sur la péninsule; un mouvement mondial d'ensemble (globalisation) auquel l'Italie doit s'adapter; l'unification monétaire et le choc que cette dernière a provoqué sur l'économie nationale. De plus, " la baisse de fonctionnalité et de prestige des

39. Sur le cas français, qui a des analogies avec l'Italie : P. Bourdieu, Homo academicus, Paris, Les Éditions de Minuit, 2002 [I984].

40. A. Rapini, "Storia, memoria e biografia degli storici ", dans A. De Bernardi, Discorso sull'antifascismo, sous la direction de A. Rapini, Milan, Bruno Mondadori, 2007, p. I-30.

4I. Il serait très utile de vérifier avec une précision empirique la réception des œuvres historiques à travers les critiques dans les revues spécialisées, dans la presse et la quantité et qualité des citations dans d'autres œuvres historiques successives.

42. E. Santarelli, Storia critica della Repubblica, ouvr. cité, p. XIV.

43. Cette expression indique la corruption politique découverte par l'enquête "Mani pulite». 
institutions italiennes constitue l'expression ultime d'une crise sociale et morale complexe et profonde ", qui puise ses racines dans la structure de la société, dans le caractère restreint des groupes dirigeants, dans leur déficit éthico-politique et dans leur manque d'hégémonie. Enfin, on assiste à une crise de la nation, qui se manifeste par l'éclosion des identités locales, l'augmentation de la Lega Nord, et l'apparition de phénomènes de racisme qui suivent les premiers flux migratoires importants.

En 1994, après l'implosion de « l'arc constitutionnel », un parti (Forza Italia) créé en quelques mois par le chef de Fininvest, Silvio Berlusconi, gagne les élections, en se présentant comme le plus fidèle interprète de l'innovation et de la rupture par rapport au passé, en tant qu'entrepreneur extérieur à la politique. Ce qui, pour certains, semble une sortie de la crise et l'arrivée d'une "Seconde République » après le tarissement de la " Première », s'avère être, au contraire - selon Santarelli - une transformation de caméléon, ou plutôt, pour utiliser un terme gramscien, une " révolution passive " : un changement purement politique dans lequel de nouveaux sujets modérés s'affirment en favorisant un plan d'ingénierie institutionnelle aux dépens d'un discours sur les réformes. La nouvelle question sociale reste en dehors de l'agenda politique, ce qui illustre parfaitement cette révolution passive. Le chômage en augmentation, la désagrégation civique de régions entières, la croissance de la pauvreté et de l'évasion fiscale, enfin, la recrudescence de la criminalité organisée, liée désormais avec la politique, se développent pourtant au cours de cette période.

Dans cette perspective - poursuit Santarelli - le vote du 27-28 mars 1994 marque la réagrégation de catégories entières qui sortent des partis traditionnels pour aller vers d'autres partis, dont Forza Italia en particulier ${ }^{44}$. Toutefois, la " révolution ", bien que "passive ", est seulement provisoire - selon l'auteur -, car le nouvel équilibre est instable et laisse présager d'autres aménagements. D'un côté, « la bourgeoisie professionnelle prend le pouvoir au sein d'un parlement rénové à plus de $70 \%$ (le nombre des entrepreneurs triple), un bloc anti-état-providence se forme, s'inspirant de ce qui s'était passé dans d'autres démocraties industrielles, [...] un bloc des classes moyennes et moyennes élevées, ouvert au grand capital et à certaines couches populaires». D’un autre côté cependant, la coalition politique, expression du nouveau bloc social, est faible (Berlusconi est allié avec Fini

44. Santarelli se sert de l'article d'Alfio Mastropaolo, «Le elezioni politiche del marzo 1994. Vecchio e nuovo nel Parlamento italiano ", Italia contemporanea, n 196, 1994, p. 46I-470. Mastropaolo a écrit sur la crise Antipolitica all'origine della crisi italiana, Naples, L'ancora del Mediterraneo, 2000. 
dans le sud et avec Bossi dans le nord) et l'implantation de la gauche est encore importante :

La complexité de la dynamique italienne [...], semble être restée à mi-chemin. Mais les contradictions étaient probablement inhérentes à une position trop formaliste et " politicienne » du processus réformateur qui avait eu lieu sans vigueur pendant au moins quinze ans, pour ensuite prendre de la vitesse sur la vague des scandales et sous l'impulsion judiciaire ${ }^{45}$.

Les années quatre-vingt de Santarelli se situent donc entre deux crises qui présentent deux physionomies principales, derrière lesquelles se trouvent de nombreux problèmes : la crise économico-financière de 1973-1975, et la crise 1992-1994. Au milieu, passe la tentative avortée de réformer le pays, d'un point de vue institutionnel ${ }^{46}$ surtout, et l'accumulation des problèmes jusqu'au crack.

En même temps que la rédaction de Storia critica della Repubblica, Pietro Scoppola (1926-2007), un intellectuel influent dans différents milieux culturels et politiques, se mesure avec l'histoire de l'Italie du temps présent. En vérité, la première édition de La Repubblica dei partiti sort en I99I, lorsque la " grande avalanche " doit encore se détacher complètement, pour utiliser la métaphore de l'historien Luciano Cafagna, qui cherche à dépeindre efficacement la crise italienne ${ }^{47}$. Une avalanche qui, bien entendu, n'était pas un événement naturel imprévisible, mais la conséquence évitable des politiques des classes dirigeantes. Peu de temps après, la quantité et la qualité des faits qui ont eu lieu entre temps oblige Scoppola à remettre à jour le livre. En 1997, le chapitre x est réécrit ainsi que deux nouveaux chapitres

45. E. Santarelli, Storia critica della Repubblica, ouvr. cité, p. 333 et 338.

46. Dans la reconstruction de Santarelli, Bettino Craxi demeure un personnage décisif pour attribuer un sens à la transition de "l'ère de l'action collective " - pour utiliser le lexique de Ginsborg - à l'effondrement de la soi-disant " Première République ». Devenu secrétaire du PSI en 1976 puis président du Conseil de 1983 à 1987, Craxi peut présenter un bilan fait d'ombres et de lumières. En politique interne, parle sans doute en sa faveur la durée du gouvernement et donc la stabilité. De plus, l'inflation est ramenée à 4,6 \% et les dépenses de l'État sont remises en ordre. L'aspect négatif est représenté par le niveau exorbitant du déficit public, qui pénalise les générations suivantes (de 456 milliards en 1983 à 800 milliards en 1987), par l'échec des réformes institutionnelles, par la dépendance de la présidence du Conseil face aux pouvoirs économiques et par la base sociale restreinte du consensus. De plus, la réduction de l'inflation, avec le « ralentissement " de l'échelle mobile, provoque des coûts sociaux dont il faut tenir compte. Enfin, il faut ajouter «la vidange " de son parti à cause du plébiscitarisme autoritaire et de la corruption déferlante pour conquérir des morceaux de pouvoir. En politique extérieure, Santarelli n'emploie pas de tons aussi élogieux que d'autres observateurs. S'il est vrai que Craxi a mis un frein à la traditionnelle dépendance face aux États-Unis avec le geste symbolique de Sigonella, les signes les plus importants de sa politique extérieure demeurent contradictoires et pleins de "faiblesse organique ", car dénués d'une coordination à l'échelle européenne et d'une idée de Méditerranée à opposer à l'atlantisme. Voir E. Santarelli, Storia critica della Repubblica, ouvr. cité, p. 277-279.

47. L. Cafagna, La grande slavina: l'Italia verso la crisi della democrazia, Venise, Marsilio, I993. 
(chap. XI. La fine di un sistema politico, chap. XII. Una normalità difficile), pour un total de 90 pages. Comme on peut le déduire du premier chapitre, dans lequel réapparait le concept de normalité de Lanaro, la mise à jour a également pour but de prendre position par rapport aux interprétations avancées par d'autres d'historiens. Qui est donc l'auteur de la nouvelle synthèse?

Intellectuel de renom de l'université la Sapienza de Rome, Scoppola est un représentant du catholicisme démocratique, expression avec laquelle on désigne en Italie un bloc politique et culturel très proche de la démocratie chrétienne, sans toutefois partager ses manifestations les plus dogmatiques en matière religieuse, ni les plus anticommunistes dans ses rapports avec la Gauche. Au milieu des années soixante, par exemple, Scoppola soutient le projet de loi «Fortuna » sur le divorce, puis défend la loi à l'occasion du référendum abrogatif de 1974. En I983, au moment de l'ascension de Bettino Craxi, il devient sénateur (indépendant) dans les rangs de la démocratie chrétienne. Au début des années quatre-vingt-dix, il est l'un des principaux partisans du référendum sur l'abolition de la loi électorale proportionnelle et de "l'ajustement de la démocratie italienne à des modèles européens plus mûrs ", tels les modèles anglo-saxons ${ }^{48}$. Peu avant de mourir, il promeut la formation du parti démocratique comme lieu de rencontre des cultures réformistes catholiques, laïques et socialistes.

D'un point de vue scientifique, Scoppola s'affirme bientôt comme historien du catholicisme et de l'Église : en I96I paraît le volume Crisi modernista e rinnovamento cattolico in Italia ${ }^{49}$, en 1966 Coscienza religiosa e democrazia nell'Italia contemporanea ${ }^{50}$, en 1967 Chiesa e stato nella storia d'Italia ${ }^{51}$. Dix ans plus tard sort le célèbre La proposta politica di De Gasperi ${ }^{52}$ et en 1985 , La nuova cristianità perduta ${ }^{53}$. Parmi ses dernières contributions, consacrées au sujet de la crise italienne, comptent également 25 aprile: liberazione ${ }^{54}$ et La costituzione contesass.

La Repubblica dei partiti tourne autour de la problématique de la crise, qui entre dans le sous-titre du volume - Evoluzione e crisi di un sistema politico - et que l'auteur propose comme métaphore générale sur l'Italie, de 1945 à l'Italie berlusconienne. Quels sont les aspects de la crise dont

48. P. Scoppola, La Repubblica dei partiti. Evoluzione e crisi di un sistema politico I945-1996, ouvr. cité, p. 488.

49. Bologne, il Mulino.

50. Bologne, il Mulino.

5I. Rome-Bari, Laterza.

52. Bologne, il Mulino.

53. Rome, Studium.

54. Turin, Einaudi, 1995.

55. Turin, Einaudi, 1998. 
Scoppola retrace la généalogie? Comme nous l'explique le sous-titre, il s'agit d'une crise du système politique. Empruntée à Paolo Farneti, l'expression perd néanmoins toute référence aux forces sociales, aux cultures politiques, aux subcultures et aux conditions de développement - à la société civile - et s'applique presque exclusivement à la " société politique » et aux institutions ${ }^{56}$. Le système politique, selon Scoppola, se résume dans les partis de masse. Par conséquent, la crise se manifeste comme une crise des principaux partis politiques, qui passent du statut de constructeurs de la démocratie, lors du passage du fascisme au post-fascisme, à celui de frein, voire même d'obstacle à la démocratie entre les années quatre-vingt et quatre-vingt-dix. Cette dégénérescence commence à la fin des années soixante-dix, lorsque le PCI et la DC subissent les effets de la modernisation du pays et vivent une " crise profonde des idéologies ${ }^{57}$ ". Le premier subit la décomposition de son bloc social, déboussolé par la disparition des paysans, l'érosion de la classe ouvrière et l'avancée de la classe moyenne ainsi que d'un horizon inconnu de valeurs post-matérialistes. La seconde recule face à la sécularisation et au rôle prépondérant d'une église, celle de Jean-Paul II, qui assume pleinement l'initiative politique au lieu de la laisser entre les mains du parti des catholiques. Le dogme de l'unité politique des catholiques commence à vaciller.

La crise des principaux partis populaires, qui sont le cœur du système politique, s'étend naturellement à toute l'architecture institutionnelle, en favorisant le succès des partis politiques les moins idéologiques, voire même post-idéologiques, comme le parti républican (PRI) et le parti socialiste (PSI). Les deux formations, allégées des fardeaux qui les liaient au passé, sont mieux préparées pour faire face aux défis du changement. La conquête du Viminale (présidence du Conseil des ministres) par le républicain Spadolini (198I-I983) et le socialiste Craxi (1983-1987) serait ainsi le reflet de la crise du consensus qui se faisait auparavant autour du PCI et de la DC. Selon Scoppola, c'est le début d'une période où les petits partis, sans lesquels il n'y a plus de majorité font basculer un système qui ne permet pas l'alternance des forces au gouvernement, et qui est incapable de se réformer. En d'autres termes, les forces politiques mineures parviennent à obtenir une part de pouvoir supérieure à leur consensus électoral, en vertu du chantage qu'elles exercent sur la stabilité des gouvernements. L'immobilisme devient de plus en plus évident, mais aussi la résistance à

56. Pour les définitions du système politique comme interaction entre société civile et société politique, voir P. Farneti, Il sistema politico italiano, Bologne, Il Mulino, 1973.

57. P. Scoppola, La Repubblica dei partiti. Evoluzione e crisi di un sistema politico 1945-1996, ouvr. cité, p. 407. 
tout type de changement de la part de tous ceux qui profitent. Aux discours superflus sur la nécessité d'une "réforme du réformateur » correspond un échec de tout changement concret, comme le prouve le nulla di fatto de la commission bicamérale initiée par le gouvernement Craxi.

Dans cette situation de blocage du système politique, qui « flotte sur la crise ", la dégradation de la démocratie s'accélère. Avec les gouvernements du Pentapartito, c'est-à-dire les coalitions de la fin des années quatre-vingt dominées par la triade Craxi-Andreotti-Forlani (CAF), la paralysie atteint son pic : les élections ne produisent aucun changement significatif, les partis s'éloignent de la société, les leaders sont égocentriques et la corruption galopante, les grands débats idéologiques sont remplacés par des disputes pathétiques sur la répartition du pouvoir, le déficit public augmente avec des dépenses folles et clientélistes. Enfin, les pouvoirs occultes étendent leur influence dans les espaces vides laissés par la faiblesse des institutions démocratiques ${ }^{58} »$. Puis ce sera "Tangentopoli ».

Au début des années quatre-vint-dix - écrit Scoppola - il est désormais évident pour tout le monde qu'il faut intervenir sur le système politique, en brisant le « lien entre parlementarisme et proportionnelle ", vrai responsable du manque d'alternative, de changement et donc de propositions de réforme. Le référendum abrogatif de 1993 de la loi électorale proportionnelle marque un changement historique. Dans le passage suivant, on peut observer toute la distance avec l'interprétation continuiste de Santarelli :

une longue phase de l'histoire de la République italienne s'est achevée entre deux référendums : celui du 2 juin 1946 et celui du I8 avril I993. Avec cependant une différence entre les deux : le premier est constitutif de la République, même si son appareil constitutionnel était entièrement à définir; le second brise une continuité de système, mais n'en définit pas encore un nouveau, et avec cela ouvre une transition difficile et incertaine ${ }^{59}$.

Dans le chapitre final, Una difficile normalità, Scoppola reprend le concept flou de "normalité " de Lanaro. L'auteur a la tâche difficile d'expliquer pourquoi la réforme de la loi électorale et de la constitution matérielle s'inspirant du modèle anglo-saxon ne résout pas la crise, mais ouvre plutôt une phase d'instabilité et de transition. En d'autres termes, si la source de la crise italienne se trouve dans le blocage du système politique, pourquoi les interventions sur ce terrain se révèlent-elles inefficaces? Pourquoi la " normalité » demeure-t-elle souhaitée mais inaccessible? Loin de mettre en discussion la vision politique de l'analyse, Scoppola conclut que la 
responsabilité doit être attribuée à un manque de volonté de réforme du système bipolaire et donc à " une réforme inachevée ${ }^{60}$ ":

le système hésite entre un cheminement difficile mais nécessaire vers un mécanisme plus cohérent d'alternance et un retour à la proportionnelle qui entrainerait le rétablissement d'une situation où les partis ont un rôle démesuré : la république des partis peut-elle renaître de ses cendres? [...] L'issue de secours normale de la démocratie des partis s'est avérée fondée. Les référendums électoraux et les enquêtes de la magistrature, doublés du "tremblement de terre électoral de 1992 ", ont entrainé sa chute; toutefois, les limites, les retards et les contradictions avec lesquels la réforme a été effectuée rendent possible des tentatives de restauration et par conséquent, rendent également plausible l'hypothèse d'une solution fondée sur une rupture radicale et sur la discontinuité constitutionnelle ${ }^{6 \mathrm{I}}$.

Cependant, l'interprétation de Scoppola n'examine pas les racines de Berlusconi et du berlusconisme.

\section{La transition}

Lorsqu'en 1998 Paul Ginsborg publie L'Italia del tempo presente pour étendre son Storia dell'Italia repubblicana à une période plus récente, le pays traverse une phase d'incertitude et d'instabilité politique, dont Scoppola avait prédit les aspects institutionnels et Santarelli les aspects sociaux. La victoire de Berlusconi en 1994 se révèle n'être qu'une éphémère tentative de sortir de la crise, puisque seulement huit mois plus tard, le fondateur de Forza Italia doit quitter le Viminale à cause du départ de la Lega Nord de sa coalition et d'un désaccord populaire croissant. Excluant des élections anticipées, le président de la République Scalfaro confie au " technicien » Lamberto Dini la tâche de former un gouvernement et de préparer les élections. Elles ont lieu le 2I avril I996. La première victoire de Romano Prodi et de la coalition de centre-gauche (Ulivo) avec le soutien de Rifondazione comunista bouleverse les équilibres politiques nationaux.

Cependant, la stabilisation est encore une fois fragile. Le 2I octobre 1998, Romano Prodi est désavoué par Rifondazione comunista. Avant les nouvelles élections de 200I, la législature (XIII) continue avec deux gouvernements de l'ex-communiste D'Alema et le retour du socialiste Giuliano Amato à la présidence du Conseil. L'Italie semble sortir de la crise du début des années quatre-vingt-dix, en consolidant dans un premier temps un bloc

6o. C'est le titre de l'avant-dernier paragraphe du livre.

6I. P. Scoppola, La Repubblica dei partiti. Evoluzione e crisi di un sistema politico I945-I996, ouvr. cité, p. 525. 
social et politique de droite à fortes tendances populistes puis, peu après, de centre-gauche : le siège est réciproque ${ }^{62}$. Le pays est fondamentalement divisé en deux et les deux coalitions sont faibles. Par conséquent, tout est encore possible.

Dans un tel climat, il est difficile pour Ginsborg d'identifier des tendances nettes de moyenne et longue durée. La périodisation de la matière à ordonner elle-même en subit les conséquences, reflétant les incertitudes du présent. De ce point de vue, l'essai montre plus de nuances par rapport à celui qui avait paru en 1989. Cependant, l'historien anglais fournit l'analyse la plus riche de l'Italie du temps présent, une analyse attentive à chaque aspect économique, social, politique et institutionnel du pays et à leurs liens réciproques. Dans le volume de Ginsborg, l'analyse et l'articulation importante des années quatre-vingt correspondent directement à une définition de la crise polysémique et multiple; deux chapitres denses sont consacrés à cette crise : le cinquième - Il fallimento della politica (1980I992) - et le huitième, qui conclut le livre - I tempi della crisi (I992-I996). Comment le concept de crise y est-il construit? Lauteur refuse les explications mono-causales et ajoute d'autres facteurs, de brève et de longue durée, nationaux et internationaux, liés aux vices mais aussi aux vertus des italiens.

Parmi les facteurs externes, il faut énumérer aussi bien la chute du mur de Berlin, qui, mettant fin à la guerre froide, contribue à désagréger les équilibres du " pentapartito " et à mettre en marche le système politique italien, que le processus d'unification européenne. L'unification, par exemple, impose à l'Italie des comportements économiquement vertueux, qui mettent un frein à l'usage clientéliste du déficit public. En effet, le clientélisme est utilisé traditionnellement comme un anesthésiant pour les conflits sociaux. Ces derniers, désormais, commencent à exploser.

Parmi les facteurs internes, Ginsborg énumère la dégénération du système des partis, visible dans la corruption, le clientélisme, le «familisme ", la solidarité avec la criminalité organisée, enfin dans l'inefficacité de l'administration publique. Cependant, on doit également signaler l'action positive de minorités, porteuses d'un sens élevé de l'État et de l'intégrité morale, comme les magistrats qui mettent au grand jour les rapports pervers entre politique et économie ("Tangentopoli »), ou bien les techniciens, qui œuvrent au sein des ministères pour amener les comptes publics au niveau souhaité par les directives européennes.

62. Sur le concept de populisme, voir A. Mastropaolo, La mucca pazza della democrazia: nuove destre, populismo, antipolitica, Turin, Bollati Boringhieri, 2005. 
Parmi les facteurs qui forment les divers aspects de la crise, il faut également mentionner l'émergence d'une classe moyenne individualiste et égoïste, qui se retrouve autour de phénomènes comme l'antiméridionalisme, le particularisme, le racisme, le culte d'identités locales aussi agressives qu'inventées ou encore l'hostilité envers l'imposition. Ces sentiments trouvent leur représentation politique dans la Lega Nord ${ }^{6_{3}}$. À cette nouvelle classe, s'ajoute cependant - selon Ginsborg - un autre secteur de la classe moyenne, auquel il donne le nom de "classe moyenne réflexive", non sans un soupçon d'abstraction ${ }^{64}$. Cet acteur, face aux difficultés rencontrées par le pays, se fait le chantre de solutions politiques nationales au sein d'un réformisme traditionnel, en contraste avec le localisme. Enfin, la croissance démesurée de la criminalité organisée contribue à la déflagration de la crise. Protégée par une partie de la classe dirigeante, la mafia voit son influence augmenter de manière exponentielle au début des années quatre-vingt-dix, avec l'utilisation de la «stratégie de la dynamite » et les assassinats des juges Falcone et Borsellino, sur lesquels la magistrature continue à enquêter encore aujourd'hui. Toutefois, même dans ce cas, il existe une alternative positive, qui réagit à la crise : la révélation de l'existence d'anticorps dans les strates de la démocratie (comme les réactions de la société civile, en particulier sicilienne), l'action de contraste des juges antimafia et l'apparition de forces politiques concentrées sur l'intransigeance morale. Ces éléments constituent un facteur supplémentaire de composition et d'accélération de la crise, car ils contribuent à fixer un point de non-retour relativement aux pratiques politiques les plus obscures du passé. Malgré cela, ils sont un encouragement à aller de l'avant et à couper les nœuds gordiens.

Pour conclure, à l'intérieur de cette riche architecture conceptuelle et analytique, les années quatre-vingt de Ginsborg peuvent être interprétées comme un compte-rendu sur la formation et la stratification des différents niveaux de la crise, jusqu'aux convulsions extrêmes du pays avec l'implosion des partis de l'arc constitutionnel.

Le second gouvernement Amato, qui se présente aux italiens comme un gouvernement de transition, après l'implication désastreuse de Massimo D’Alema dans la guerre des Balkans, conduit la XIII ${ }^{\mathrm{e}}$ législation à son terme. Au printemps 200I, à quelques mois du rendez-vous délicat du G8 à Gênes, prévu pour le mois de juillet, se déroulent les nouvelles élections.

63. G. De Luna (dir.), Figli di un benessere minore: la Lega 1979-1993, Scandicci, La nuova Italia, 1994. 64. Les références de Ginsborg sont : U. Beck, A. Giddens et C. Lasch, Reflexive Modernisation, Cambridge, Cambridge University Press, 1994 et S. Lash et J. Urry, Economies of signs and space, Londres, Sage, I994. 
Encore une fois, on assiste à un total renversement de situation. Le centregauche est battu et Silvio Berlusconi, allié de la Lega Nord et d'Alleanza Nazionale, retourne au Viminale. La transition italienne semble infinie et le discours politique continue à être dominé par le thème de la réforme constitutionnelle et institutionnelle nécessaire pour effectuer le passage à la prétendue "Seconde République ". Bien entendu, par transition, on entend, d'une part, la formation d'un bloc social solide, qui trouverait une expression politique stable dans une coalition, d'autre part, la sortie d'un gué intermédiaire entre l'abandon du modèle constitutionnel de 1948 - basé sur la centralité du parlement, sur la loi électorale proportionnelle et sur le rôle clé des partis de masse et des organisations syndicales - et l'affirmation d'un autre modèle (le modèle actuel) où c'est l'exécutif qui prévaut, au nom de la primauté de la "gouvernabilité ", la loi majoritaire pour les élections, la fonction charismatique du leader et la marginalisation des syndicats.

Malgré la gestion tragique du G8 de Gênes, durant lequel les carabiniers tuent le jeune Carlo Giuliani et les droits constitutionnels sont suspendus pendant trois jours de "boucherie à la chilienne ${ }^{65}$ ", le gouvernement Berlusconi non seulement tient, mais arrive jusqu'au bout de la XIV législature (2006). Il s'agit sans doute d'une nouveauté significative pour un pays habitué à la succession de gouvernements de courte durée, qualifiés, de façon fantaisiste, de "balnéaires ", " techniques ", " institutionnels ", de "garantie » et, récemment, de la "responsabilité ». Toutes ces formules masquent l'instabilité historique des exécutifs, à l'intérieur desquels, cependant, la zone de représentation politique ne coïncide pas avec la zone de légitimité politique, qui exclut les communistes du gouvernement jusqu'en I99I. Cette situation se répercute dans l'interprétation de la crise et de la transition dans la reconstruction des années I980 et ainsi que dans l'interprétation des significations profondes à lui attribuer, dans la longue durée de l'histoire nationale.

\section{Avec le gouffre derrière nous}

L'importance extrême des années quatre-vingt pour comprendre la généalogie de la crise, mais en même temps, en inversant les termes, l'importance

65. C. Antonini, F. Barilli et D. Rossi (dir.), Scuola Diaz: vergogna di stato: il processo alla polizia per l'assalto alla Diaz al G8 di Genova, Rome, Edizioni Alegre, 2009. Voir les documents judiciaires en français : <https:// www.supportolegale.org/>. 
de la crise pour comprendre les phénomènes de la décennie, devient évidente dans le champ historiographique pendant la XIV e législature.

Dans cette perspective, le colloque qui a eu lieu à Rome en 2003 et dont le titre est Gli anni Ottanta come storia, est tout à fait paradigmatique : il a la fonction d'une épiphanie. Le département d'Innovation et Société (DIeS) de l'université « La Sapienza » de Rome, la Fondazione Istituto Gramsci, l'université Suor Orsola Benincasa de Naples et la Luiss Guido Carli convoquent un groupe d'historiens "aux orientations politico-culturelles et méthodologiques différentes ", convaincus que :

la crise soudaine du système politique italien ayant explosé au début des années quatrevingt-dix peut être comprise uniquement à travers un examen approfondi, jusqu'ici insuffisant, des transformations politiques, économiques et sociales considérables qui ont touché le monde, et en partie l'Europe, au cours de la décennie précédente. [...] Pour toutes ces raisons, les années quatre-vingt peuvent être considérées charnière dans l'histoire de la seconde moitié du Vingtième siècle. Par de nombreux aspects, les intrigues des deux décennies précédentes revinrent en partie à la surface, et se résolurent dans l'arène internationale et dans la pratique politique interne, donnant lieu à de nouvelles orientations qui sont encore aujourd'hui au centre du débat politique ${ }^{66}$.

La préface aux actes du colloque, édités l'année suivante, se charge en outre de tracer les contours de la crise; de tracer également le cadre herméneutique au sein duquel s'insèrent les contributions des différents historiens : " de fortes impulsions au changement, mais également des résistances tenaces des apparats traditionnels par rapport aux problèmes émergeants de la politique extérieure et intérieure caractérisent les années 80 ». Puis elle continue en concluant qu' " en réalité, l'histoire de la décennie a été largement traversée par la crise des partis traditionnels de la République et qu'elle en a surtout accéléré la fin ${ }^{67}$ ".

En accord avec cette vision des choses, on peut lire l'intervention de Gaetano Quagliariello. Originaire de Naples et lié à la tradition libérale, il milite dans le parti radical jusqu'au début des années quatre-vingt, en tant que vice-secrétaire national. Il s'inscrit immédiatement à Forza Italia au moment de la naissance du parti de Berlusconi, et peu après, il collabore avec "Ideazione ", la revue théorique du parti. En 2006, il entre au parlement avec Berlusconi, parmi les sénateurs, et l'année suivante, il est responsable du département Culture de Forza Italia. En 2008, il est réélu sénateur et il occupe actuellement le poste de vice-président adjoint du Popolo della libertà ${ }^{68}$.

66. S. Colarizi et al., Prefazione a Gli anni Ottanta come storia, Rubbettino, Soveria Mannelli, 2004, p. 7.

67. Ibid., p. Iо.

68. <http://www.gaetanoquagliariello.it/node/72>. 
Quand Quagliariello participe au colloque romain, il est conseiller aux affaires culturelles du président du Sénat Marcello Pera et il contribue presque en même temps à la création de la Fondation Magna Carta, "un nom évocateur qui contient en même temps la défense de la tradition, sans préjugés contre les changements imposés par la modernité ${ }^{69}$ ". Magna Carta, comme le dit son président Gaetano Quagliariello, est " un lieu de formation et de recherches d'inspiration libérale sans aucune soumission culturelle ni engagé avec le centre droit $^{70}$.

D'un point de vue académique, l'historien de Naples est professeur à l'université privée Luiss "Guido Carli » où, à l'époque de la publication de Gli anni Ottanta come storia, il dirige le département de sciences historiques et socio-politiques. Son principal objet d'étude est De Gaulle, auquel il a consacré une biographie politique, parue la même année que le colloque romain ${ }^{71}$.

Ces informations, destinées à situer l'historien biographiquement, culturellement, politiquement et institutionnellement, rendent son intervention bien plus compréhensible. Quagliariello met de côté le concept de crise pour décrire l'Italie du temps présent, car - selon lui - le pays serait désormais entré dans une phase de stabilisation. Pour utiliser une métaphore, il serait sorti du cône d'ombre de la crise et entré dans le cône de lumière du berlusconisme. L’intervention est donc une généalogie du phénomène politique Berlusconi, une recherche de ses racines dans les dynamiques des décennies précédentes. Dans cette perspective, les années quatre-vingt ont un rôle clé. Elles sont une sorte de charnière dans la mesure où, d'une part, elles mettent fin à l' "ancien régime » - dit l'auteur - mais d'autre part elles produisent les premières bases de la révolution libérale mise en œuvre par Berlusconi et permettent les conditions pour l'affirmation de la liberté. La transition est donc antidatée d'environ une décennie. Quagliariello affirme avec un ton gramscien :

les années quatre-vingt, du point de vue politico-institutionnel, doivent être considérées comme une période typique de transition : l'ancien régime reste formellement en vigueur, mais les éléments qui en provoqueraient l'écroulement se trouvent en son sein. Ancien et nouveau s'hybrident, en créant dans l'Histoire de l'Italie républicaine une sorte de flou, dans lequel l'ancien équilibre apparaît désormais inévitablement dépassé, cependant qu'une nouvelle réalité peine à s'affirmer ${ }^{72}$.

69. <http://www.magna-carta.it/content/collaboratori >

70. <http://www.gaetanoquagliariello.it/node/72>.

71. De Gaulle e il gollismo, Bologne, Il Mulino, 2003.

72. G. Quagliariello, « Gli anni Ottanta: gli aspetti politico istituzionali. Un'interpretazione », dans S. Colarizi et al., Gli anni Ottanta come storia, ouvr. cité, p. 267. 
Quand la décennie de transition commence-t-elle? Quagliariello utilise une périodisation longue pour classifier les années quatre-vingt. Il situe le terminus a quo précisément en 1978. C'est à ce moment qu'on perçoit les signaux de fléchissement du "vieux ». L'assassinat d'Aldo Moro de la part des Brigades rouges met une croix définitive sur le « compromis historique ", c'est-à-dire sur l'idée de faire de l'alliance entre PCI et DC l'axe du système politique. De toute manière, au-delà des divergences entre les deux partis de masse, ceux-ci allaient cesser d'être les principaux protagonistes de la scène politique. Avec les élections administratives de 1978 commence la lente et constante baisse électorale du PCI. La même année, le référendum contre la loi Reale, proposé par le parti radical, bien que rejeté, démontre cependant l'existence d'une partie consistante du pays $(23,3 \%)$ qui échappe aux directives des principaux partis. De plus, 1978 est l'année de la démission du président de la République Leone, contraint à ce geste à cause d'une « campagne de presse » où Quagliariello entrevoit les signes avant-coureurs de futures campagnes médiatiques contre des personnalités de la politique. La démission de Leone, au-delà des modalités de son développement, signifie tout de même la " certification » de l'existence d'une "questione morale ", qui aura de graves répercussions sur la stabilité du système politique. Enfin, 1978 est l'année du socialiste Sandro Pertini, élu nouveau président de la République. Pertini, qui reste dans le cœur des Italiens pour son humanité et son harmonie avec l'humeur du pays, crée un style présidentiel interventionniste et « charismatique ». Il est à la base d'une césure dans la conception de la présidence « vue comme élément d'équilibre face aux interna corporis des institutions. Avec Pertini - poursuit Quagliariello - une époque fut inaugurée, dans laquelle le Président passerait de chef d'orchestre discret à acteur protagoniste de la vie politique, en contact direct et privilégié avec l'opinion publique ${ }^{73}$ ».

Pour toutes ces raisons, «l'équilibre institutionnel fondé sur la centralité des partis a subi [...] un coup dur ${ }^{74}$ ». Une phase d'incertitude s'ouvre alors. Elle se prolonge jusqu' en I992, terminus ad quem des « longues années 80 ", dont la preuve est visible au centre du débat politique dans la substitution du thème de la "Constitution à appliquer " avec celui de la "Constitution à réformer ". En 1992, le système implose. Cette déferlante représente l'échec de la tentative de réformer "par des lignes internes l'ancien régime 
qui s'éteint définitivement. En même temps, le processus de liquidation des partis classiques s'est accéléré et a pris directement la voie judiciaire ${ }^{75}$ ».

Dans la vision de Quagliariello donc, la " décennie longue » est d'un côté l'histoire des tentatives avortées d'auto-réforme du système politicoinstitutionnel; d'un autre côté, il s'agit de la réalisation de certaines anticipations «qui se seraient mises en place après l'implosion du système ${ }^{76}$ " avec Berlusconi deus ex machina de la nouvelle Italie. Parmi celles-ci, les plus importantes sont le renforcement de l'exécutif, et, en son sein, l'exercice d'un plus grand leadership du président du Conseil, non seulement au détriment du parlement et des autres forces politiques, mais aussi et surtout au détriment des forces syndicales et de leur " pouvoir de veto " sur la politique économique. Le modèle explicite d'une telle pratique de gouvernement est le "décret de la saint Valentin " de 1985, avec lequel Bettino Craxi baisse de quatre points l'échelle mobile sur les salaires avec le seul accord des syndicats CISL e UIL ${ }^{77}$.

Lorsque la XIV éégislature s'achève de "façon naturelle », les élections législatives de 2006 semblent démentir l'interprétation de Quagliariello sur la fin de la transition et provoquent un nouveau bouleversement : les italiens préfèrent Romano Prodi et la coalition de centre-gauche (l'Unione) à la Casa delle libertà. En réalité, le Pays est de nouveau divisé en deux et la victoire parlementaire n'est rendue possible que grâce à une loi électorale discutable. Il suffit de dire qu'à la Chambre des députés, le centre-gauche a une majorité de seulement 25224 voix et qu'au Sénat elle a d'environ 200000 voix en moins que le PDL (Popolo delle Libertà), la Ligue du Nord et le Mouvement pour les Autonomies. Au bout de deux ans, le second gouvernement Prodi s'effondre à cause de sa faiblesse électorale et politique. On retourne aux urnes et Berlusconi parvient encore une fois à interpréter l'humeur de la majorité des italiens : sa victoire au Parlement est écrasante. Pour la première fois dans l'histoire de la République, les socialistes et les communistes disparaissent de la Chambre et du Sénat ${ }^{78}$. Dans ce climat politique qui semble démontrer l'importance du consensus berlusconien et l'enracinement du soi-disant berlusconisme dans de nombreux

75. Ibid., p. 27 I.

76. Ibid., p. 280.

77. Sur ce thème on peut voir S. Colarizi, «La trasformazione della leadership. Il PSI di Craxi (1976-I98I) ", dans S. Colarizi et al., Gli anni Ottanta come storia, ouvr. cité, p. 3I-64.

78. Pour les résultats détaillés, consulter le site officiel du ministère de l'Intérieur : <http://elezionistorico. interno.it/>. 
secteurs sociaux et culturels du pays, parait Autobiografia di una Repubblica. Le radici dell'Italia attuale 79 .

L'auteur, Guido Crainz, vient de l'expérience de la New Left italienne, au sein de laquelle il a milité dans les rangs de Lotta continua. Bien qu'on ne connaisse pas réellement ses positions politiques actuelles, on peut sans nul doute le ranger dans le vaste " territoire » de la Gauche, à laquelle il appartient toujours, contrairement à beaucoup de représentants des mouvements des années 1960-1970, qui s'en éloignèrent parfois avec des gestes symboliques spectaculaires. Crainz est un universitaire qui enseigne l'histoire contemporaine à la faculté de sciences de la communication de l'université de Teramo. Ses recherches commencent avec les ouvriers agricoles de la plaine du Po' (Padania. Il mondo dei braccianti dalla fine dell'Ottocento alla fuga delle campagne ${ }^{80}$ ) et se poursuivent principalement avec l'histoire de l'Italie républicaine (Storia del miracolo italiano ${ }^{8 \mathrm{r}}$;l paese mancato. Dal miracolo economico agli anni Ottanta ${ }^{82}$; Il dolore e l'esilio. L'Istria e le memorie divise d'Europa ${ }^{83}$ ).

L'un des traits distinctifs de sa façon d'interpréter la présence de l'historien dans la société est sa sensibilité pour les territoires intermédiaires entre l'histoire (historia rerum gestarum) et les usages publics de l'histoire, qui définissent un espace aux frontières extrêmement variables, où il arrive souvent de se retrouver face à des manipulations et à des instrumentalisations politiques du passé ${ }^{84}$. Crainz tente de parcourir cet espace, où les moyens de communication jouent un rôle essentiel, avec l'ambition de parler à un public plus vaste que celui de la communauté scientifique, sans pour autant sacrifier la complexité du langage et de la pratique de l'historien. Dans ce même esprit, on se doit de rappeler les collaborations de Crainz avec les quotidiens la Repubblica, il Messaggero Veneto, Il Piccolo, avec la radio Radio Tre e la télévision public Rai Tre ${ }^{85}$, ou bien encore la direction des archives de la mémoire des Abruzzes, où l'on recueille des films de famille. L'Autobiografia di una Repubblica - livre que l'on ne peut

79. G. Crainz, Autobiografia di una Repubblica. Le radici dell'Italia attuale, ouvr. cité.

8o. Rome, Donzelli, I994.

8I. Rome, Donzelli, I997.

82. Rome, Donzelli, 2003.

83. Rome, Donzelli, 2005 .

84. Sur le même thème, on renvoie à N. Gallerano, Le verità della storia: scritti sull'uso pubblico del passato, Rome, Manifestolibri, 1999 et B. Groppo (dir.), Historiens et usages publics du passé. Allemagne, Italie, Russie, Matériaux, $\mathrm{n}^{\circ}$ 68, 2002.

85. Pour la télévision, il a réalisé les programmes suivants : Gli anni cinquanta (avec Corrado Farina), vingt épisodes, Raieducational 1997; Il paese mancato (avec Italo Moscati), dans la série « La grande Storia in prima serata ", Raitre 2005 . 
passer sous silence - est un texte double, qui examine prudemment l'historiographie en regardant le présent, le positionnement du texte dans le discours public et, évidemment, ses effets.

Le volume commence avec une série de questions tranchantes : l'Italie est-elle encore dans une phase de transition suite à la crise du début des années quatre-vingt-dix? Le succès de Berlusconi constitue-t-il une étape supplémentaire de cette transition? S'agit-il d'un succès éphémère, dû à la force des télévisions, à la fascination du populisme, à l'inconsistance des oppositions, ou bien s'agit-il d'une stabilisation qui repose sur quelque chose de plus profond, et donc de plus long? La thèse soutenue par l'auteur est tout aussi tranchée : il s'agirait de la seconde hypothèse. En Italie, « une mutation anthropologique " s'est opérée, un changement dans la façon d'être des Italiens, de leurs rêves et de leurs désirs ${ }^{86}$. La crise du début des années quatre-vingt-dix est certainement une crise du système politique; cependant, derrière elle, s'étendent des processus de longue durée qui permettent à Berlusconi de gagner les élections de 1994 avec une rapidité foudroyante. Le rôle de l'historien est de comprendre le quand et le comment de tels processus.

Si la thèse de la stabilisation rappelle celle que défendait Quagliariello quelques années auparavant, sa démarche en est en revanche beaucoup plus éloignée. Crainz se base en effet sur la culture en tant qu'ensemble des styles de vie, des façons de consommer, des représentations et autoreprésentations, s'appuyant surtout sur les domaines littéraires et journalistiques, qui constituent ses sources principales pour raconter la " grande transformation » des italiens.

Crainz situe l'amorce du processus annoncé durant le " miracle économique ", car c'est à ce moment précis que s'opère une rupture historique, qu'on peut rendre par l'image du changement de peau et d'âme de la péninsule. Les classes dirigeantes auraient pu et dû guider le changement, le contrôler, en s'intéressant à l'utilité publique. Au contraire, les projets réformistes et planiste de correction des altérations provoquées par le " miracle », ainsi que de celles qui lui préexistaient, échouent à cause de l'opposition d'une série de forces, dont font partie l'Église, les États-Unis, une partie des entrepreneurs, de l'apparat de l'État et les partis du gouvernement eux-mêmes. Crainz cite comme exemple le sabotage des réformes urbanistiques et fiscales, fondamentales pour protéger le paese-paesaggio ainsi que la redistribution de la richesse. L'absence de règles collectives 
l'emporte. Les particularismes et les corporatismes triomphent. Le fondateur du journal la Repubblica est appelé à décrire le passage de phases en ces termes :

quel a été le moment où une société laborieuse et dynamique s'est transformée en une immense vermine, collectivement vouée à la dilapidation des ressources et à un système de fraudes devenu système? La grande mutation génétique se situe à la fin des années soixante et coïncide avec la première véritable phase de bien-être que notre pays n'ait jamais connu. Il aurait fallu une classe dirigeante moralement et professionnellement capable d'utiliser cette richesse pour construire une société juste, civile et prospère. Nous avons au contraire participé à un grand gavage au cours duquel toutes les valeurs se sont volatilisées, toutes les règles ont été piétinées et tous les rapports ont empirés ${ }^{87}$.

À court terme, le changement est sous-estimé, car il touche des comportements qui investissent surtout la vie privée, invisibles et donc difficiles à évaluer immédiatement. De plus, la vague de longue durée de 1968 tente de contraster les valeurs dont parle Scalfari. Toutefois, sans l'intervention du sommet des classes dirigeantes, sans la création de règles fixes et partagées il est impossible de former des anticorps contre les modalités de la modernisation italienne. "Les années 68 " commencent avec la chanson Volare de Modugno (1958) et s'achèvent avec la Fièvre du samedi soir (1977). Par conséquent, lorsqu’à la fin des années soixante-dix la vague de 68 se retire, le ressac laisse derrière lui l'ensemble des particularismes produits par le miracle et par son non-gouvernement : ainsi furent engendrées les années quatre-vingt ${ }^{88}$. Pier Paolo Pasolini - selon Crainz - comprend avec lucidité le côté obscur du changement, en commentant l'apparente victoire du référendum sur le divorce en 1974 :

mon opinion est que $59 \%$ de "non " ne démontre pas miraculeusement une victoire de la laïcité, du progressisme, de la démocratie : rien de tout cela. Cela démontre au contraire que les classes moyennes ont radicalement, anthropologiquement changé : leurs valeurs positives ne sont plus celles sanfédistes et cléricales, mais sont devenues les valeurs de l'idéologie hédoniste de la consommation et l'acceptation moderniste du style de vie américain [...]. Le " non " a été sans nul doute une victoire. Mais l'indication qu'elle donne est celle d'une "mutation " de la culture italienne : qui s'éloigne autant du fascisme traditionnel que du progressisme socialiste ${ }^{89}$.

Après l'ivresse de la participation et du public des années soixante-dix, la décennie suivante, bien décrite dans le roman Altri libertini de Pier

87. E. Scalfari, dans la Repubblica du I6 janvier 1994.

88. Notons la convergence avec la thèse soutenue par P. Ginsborg, Storia d'Italia dal dopoguerra ad oggi, ouvr. cité, p. 576.

89. P. P. Pasolini, dans le Corriere della sera du ıo juinı974. 
Vittorio Tondelli ${ }^{9 \circ}$, renverse les échelles de priorité et célèbre le triomphe du privé, qui ouvre symboliquement et chronologiquement le changement de phase. Mode, divertissement, corps, individualisme, consommations privées, argent facile sont - comme l'affirme Crainz - les mots qui connotent le mieux les années quatre-vingt, dont l'univers de valeurs, de modèles culturels et de comportement est construit et véhiculé avant tout par la télévision et par la publicité. On comprend alors pourquoi le plus grand entrepreneur télévisuel a pu faire son apparition durant cette décennie, se transformant en homme politique au début des années quatre-vingt-dix. Il n'est pas anodin que cet entrepreneur voie sa fortune se décupler grâce à la solidarité de Bettino Craxi, qui est le plus fidèle interprète des années quatre-vingt, celui qui exprime le mieux les temps de la fusion perverse entre modernisation, illégalisme, corruption, clientélisme et arrogance.

Les années quatre-vingt de Crainz ne sont donc pas uniquement insérées entre les années soixante-dix et quatre-vingt-dix, mais aussi entre le miracle économique et la stabilisation berlusconienne, qui est durable, car insérée dans la mutation anthropologique de moyenne durée. Un rapport du Censis de 198I décrit les caractéristiques inquiétantes des nouveaux italiens :

la violence des comportements est étroitement liée à l'apparition d'une sorte d' "individualisme protégé » : d'un côté, on veut la liberté de déploiement des comportements individuels et collectifs la plus large possible, d'un autre côté on demande une protection publique totale [...]. Le summum de l'individualisme et le summum de la protection, presque une société de la besace, du sac à deux poches, toutes les deux entièrement pleines. Mais une telle tendance est-elle acceptable sans risquer de perdre le sens des responsabilités, dans l'illusion que tout est possible, quoi qu'il arrive ${ }^{91}$ ?

Silvio Berlusconi se chargera de donner une réponse. Les pages de Crainz nous poussent à croire que pour le patron de Mediaset, une telle tendance n'est pas seulement acceptable, mais qu'elle est même absolument souhaitable. 
